\title{
PEEP: dos lados de la misma moneda
}

PEEP: two sides of the same coin

PEEP: duas faces da mesma moeda

Orlando Rubén Pérez Nieto, * Eder Iván Zamarrón López, ${ }^{\ddagger}$ Manuel Alberto Guerrero Gutiérrez,§

Ernesto Deloya Tomas, ${ }^{*}$ Raúl Soriano Orozco, " Jesús Salvador Sánchez Díaz, " Miguel Ángel Martínez Camacho, ${ }^{\star *}$

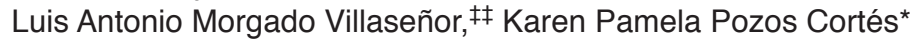

\section{RESUMEN}

Por eones, el estudio sobre el manejo de la presión positiva al final de la espiración (PEEP) ha sido exponencial y fundamental para el estudio de patologías pulmonares, sobre todo debido a su relación directa con el síndrome de dificultad respiratoria aguda (SDRA) presentando cambios y avances en las metas de protección pulmonar incluyendo volumen corriente $(\mathrm{Vt})$ bajo, presión plateau ( $\mathrm{Pp}$ ) debajo de $30 \mathrm{cmH}_{2} \mathrm{O}$, presión máxima de la vía aérea ( $\mathrm{Paw}$ ) menor a $35 \mathrm{mmH}_{2} \mathrm{O}$, presión de distensión $(\Delta \mathrm{P})$ menor a $13 \mathrm{mmH}_{2} \mathrm{O}$ y aplicación de PEEP mayor a $5 \mathrm{cmH}_{2} \mathrm{O}$, logrando disminuir la mortalidad de esta entidad. La aplicación de PEEP implica un reto al clínico, su objetivo es llevar al paciente al punto óptimo de éste, logrando la apertura de la mayoría de los alveolos y disminuyendo la lesión pulmonar inducida por el ventilador (VILI), repercutiendo a su vez en los demás órganos y sistemas.

Palabras clave: SDRA, presión de distensión, distensibilidad, PEEP, lesión pulmonar inducida por el ventilador.

\section{ABSTRACT}

For eons, the study on the management of positive end-expiratory pressure (PEEP) has been exponential and fundamental for the study of pulmonary pathologies, mainly due to its direct relationship with acute respiratory distress syndrome (ARDS) presenting changes and advances in pulmonary protection goals including low tidal volume $(\mathrm{Vt})$, plateau pressure $(\mathrm{Pp}$ ) below $30 \mathrm{cmH}_{2} \mathrm{O}$, maximum airway pressure (Paw) less than $35 \mathrm{mmH}_{2} \mathrm{O}$, distention pressure $(\triangle P)$ less than $13 \mathrm{mmH}_{2} \mathrm{O}$ and application of PEEP greater than 5 $\mathrm{CmH}_{2} \mathrm{O}$, reducing the mortality of this entity. The PEEP application involves a challenge to the clinician, its objective is to take the patient to the optimal point of this, achieving the opening of most alveoli and decreasing the ventilator-induced pulmonary injury (VILI), in turn affecting the others organs and systems.

Keywords: ARDS, driving pressure, compliance, PEEP, ventilator induced lung injury.

\section{RESUMO}

Por eras, o estudo do manejo da pressão positiva no final da expiração (PEEP) tem sido exponencial e fundamental para o estudo das patologias pulmonares, principalmente por sua relação direta com a síndrome do desconforto respiratório agudo (SDRA) apresentando mudanças e avanços nas metas de proteção pulmonar, incluindo baixo volume corrente $(V t)$, pressão de platô (Pp) abaixo de $30 \mathrm{cmH}_{2} \mathrm{O}$, pressão máxima das vias aéreas (Paw) menor que 35 $\mathrm{mmH}_{2} \mathrm{O}$, pressão de distensão (PD) menor que $13 \mathrm{mmH} \mathrm{O}_{2}$ e aplicação de $P E E P$ maior que $5 \mathrm{cmH}_{2} \mathrm{O}$, reduzindo a mortalidade desta entidade. A aplicação da PEEP implica um desafio ao clínico, seu objetivo é levar o paciente ao ponto ideal deste, conseguindo a abertura da maior parte dos alvéolos e reduzindo a lesão pulmonar induzida pelo ventilador (LPIVM), afetando por sua vez os demais órgãos e sistemas.

Palavras-chave: SDRA, pressão de distensão, distensibilidade, PEEP, lesão pulmonar induzida por ventilador.

* Hospital General San Juan del Río. Querétaro, México.

‡ Hospital CEMAIN Tampico. Tamaulipas, México.

$\S$ Instituto Nacional de Cancerología. México.

I Unidad Médica de Alta Especialidad T1. León, Guanajuato.

" Unidad Médica de Alta Especialidad No. 189 del Instituto Mexicano del Seguro Social, Veracruz, Veracruz.

** Hospital Materno-Infantil de Querétaro. Universidad Autónoma de Querétaro. México.

执 Unidad Médica de Alta Especialidad No. 71, IMSS. México.

Recibido: 09/12/2019. Aceptado: 21/01/2020.

Citar como: Pérez NOR, Zamarrón LEI, Guerrero GMA, Deloya TE, Soriano OR, Sánchez DJS, et al. PEEP: dos lados de la misma moneda. Med Crit. 2021;35(1):34-46 https://dx.doi.org/10.35366/99152

www.medigraphic.com/medicinacritica

\section{INTRODUCCIÓN}

A finales de la década de 1930, Barach y colaboradores comienzan a estudiar los efectos de la presión positiva al final de la espiración (PEEP) en ventilación mecánica en modelos animales experimentales con la presencia de sepsis, edema pulmonar y en siete pacientes humanos con falla cardiaca. ${ }^{1}$ Cournand y su equipo en 1946 miden los efectos hemodinámicos causados por la presión positiva intratorácica. ${ }^{2}$ Fue hasta 1960 cuando se empezó a incluir en la práctica clínica. Gregory y colaboradores comenzaron a aplicar PEEP a neonatos con dificultad respiratoria por déficit de surfactante, sin reporte de problemas hemodinámicos. En el mismo periodo Ashbaugh y su grupo describían el que hoy conocemos como síndrome de dificultad respiratoria aguda (SDRA) con una analogía con la dificultad respiratoria del recién nacido. ${ }^{3}$ Desde entonces, el estudio de la PEEP y del SDRA ha crecido exponencialmente acompañado uno del otro. Suter propone el aporte clínico más importante del PEEP como parte de la mejora en el transporte de oxígeno por medio de la difusión y su importancia en la mecánica ventilatoria. ${ }^{4}$ Kirby y su equipo proponen el uso de «súper PEEP», que consiste en poner PEEP hasta $25 \mathrm{cmH}_{2} \mathrm{O}$ para abrir el pulmón de esa manera, mientras tanto en Francia, Tenaillon propone que un incremento del PEEP ocasionará una disminución de $10 \%$ del retorno venoso ${ }^{5}$ y Dantzker menciona que el mecanismo primario del PEEP es optimizar la oxigenación pero disminuye el gasto cardiaco, ${ }^{6}$ Lemaire y colaboradores mencionan en ese tiempo que el retorno venoso, gasto cardiaco y PEEP están íntimamente relacionados. 7,8 Trembay describe que la reacción inflamatoria mediada por citocinas es prevenida por el uso de PEEP, Mead describe el atelectrauma, que fue ilustrado por Lachman y Ranieri. 9,10 Llegando a la década de los 90, arribó una tendencia por el uso de PEEP, Webb y Tierney mencionan que el PEEP no debería ser considerado para mejorar la oxigenación, sino como una medida de protección pulmonar; con base en esto, debemos considerar el impacto del PEEP en todos sus aspectos sobre la hemodinamia y su impacto en el organismo. ${ }^{11}$ 
Dentro de las estrategias que se han empleado con la intención de lograr una disminución en la mortalidad de los pacientes con patología pulmonar está la aplicación de PEEP, lo cual se ha establecido como una meta de protección alveolar, al prevenir la presencia de atelectrauma, definido por el daño producido por el mecanismo cíclico de cierre y reapertura alveolar cíclico, ${ }^{12}$ así como lograr el punto de histéresis pulmonar para lograr un mayor reclutamiento alveolar y aumentar el área funcional de la membrana alveolo-capilar, mejorando el intercambio de oxígeno $\left(\mathrm{O}_{2}\right)$ y dióxido de carbono $\left(\mathrm{CO}_{2}\right) \cdot{ }^{13}$

El objetivo de este artículo es realizar un análisis sobre el PEEP y sus efectos en la economía del paciente como son el sistema cardiovascular, sistema respiratorio, en los síndromes pleuropulmonares, diafragma, presión intraabdominal (PIA), sistema linfático y sobre la presión intracraneal, su uso en quirófano, pacientes con obesidad, embarazadas, indicaciones del ZEEP y el paciente pediátrico.

\section{DESARROLLO}

El objetivo del uso de PEEP es mejorar la oxigenación, sin embargo, una consecuencia natural es su posibilidad de disminuir el gasto cardiaco al aumentar la impedancia venosa por incremento en la presión positiva intratorácica de forma continua, se ha descrito que el mejor transporte de $\mathrm{O}_{2}$ variable que asocia la oxigenación y el gasto cardiaco es alcanzado por el PEEP que provee la mejor distensibilidad del sistema respiratorio (Crs), ${ }^{8}$ considerando el concepto de mejorar la oxigenación al evitar el colapso alveolar intratidal cíclico, comúnmente llamado atelectrauma, con el concepto de «abrir el pulmón y mantenerlo abierto» (OLA, open lung approach) $;{ }^{14}$ sin embargo, un estudio reciente demostró que el reclutamiento alveolar agudo con la aplicación de una PEEP titulada por Crs se asocia a mayor mortalidad comparándolo con el nivel de PEEP que corresponde por tabla de PEEP bajo/FiO 2 del grupo ARDS Network. ${ }^{15}$

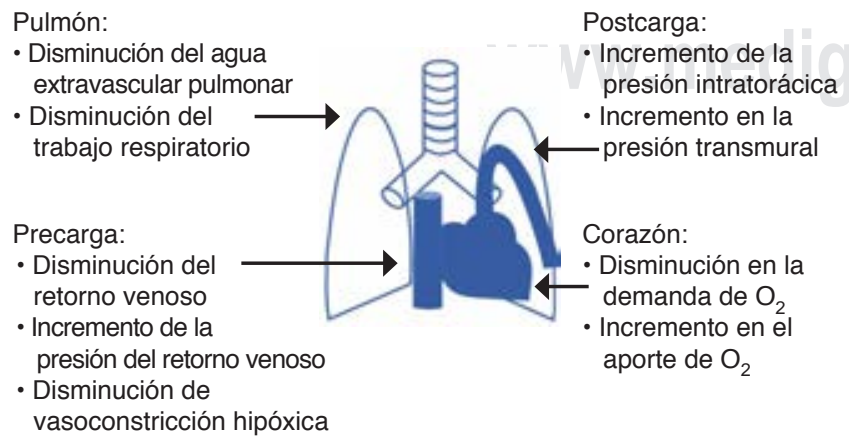

Figura 1: Efectos de una PEEP estándar sobre sistema cardiovascular.

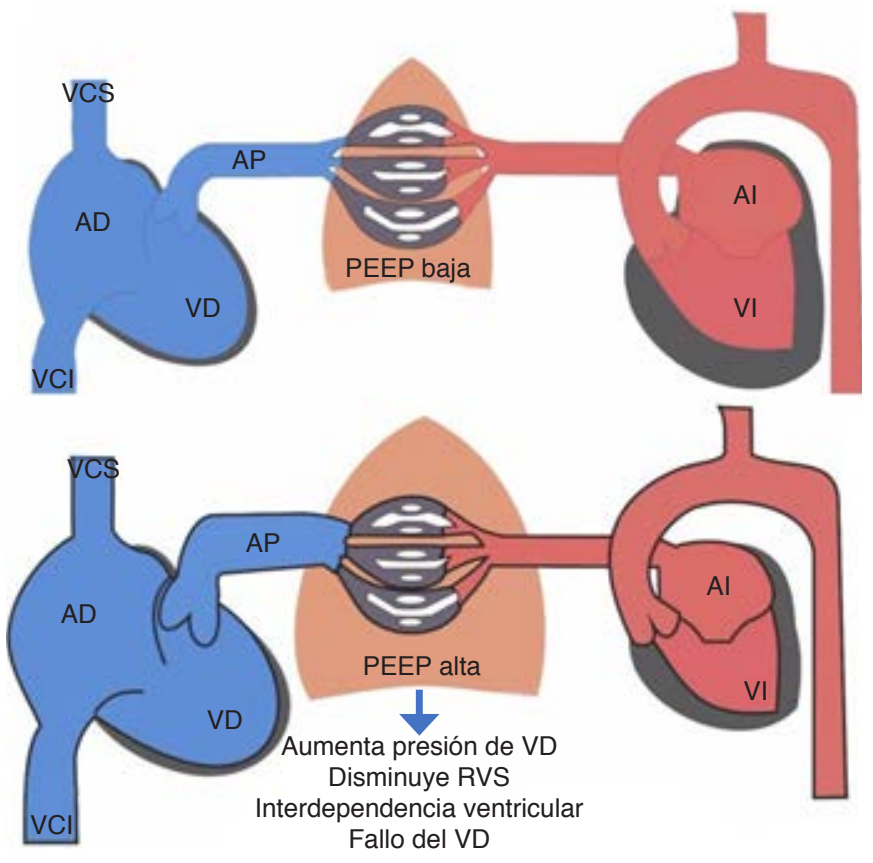

Figura 2: Efectos del PEEP sobre el ventrículo derecho.

$\mathrm{VCS}=$ vena cava superior, $\mathrm{VCl}$ = vena cava inferior, $\mathrm{AD}$ = aurícula derecha,

VD = ventrículo derecho, AP = arteria pulmonar, RVS = resistencias vasculares sistémicas, $\mathrm{VI}=$ ventrículo izquierdo, $\mathrm{Al}$ = aurícula izquierda.

Idea original: Manuel Guerrero

A lo largo de las últimas décadas, se han implementado numerosas formas de seleccionar el nivel de PEEP para un paciente con SDRA, ${ }^{16}$ dentro de las cuales se han descrito el método de la súper jeringa, guiado por el protocolo de ARDS Network, titulación por mejor Crs, por curva cuasiestática de presión/volumen, medición de presión esofágica, así como bajo guía imagenológica, ultrasonografía pulmonar o tomografía por impedancia eléctrica; sin embargo, se ha llegado a la conclusión de que la «mejor PEEP» no existe, sino que se debe buscar la «PEEP óptima» para el paciente, evaluando las diferentes variables de oxigenación, estado hemodinámico, apertura y cierre intratidal y distensibilidad pulmonar; se propone un nivel de PEEP de 5 a $15 \mathrm{mmH}_{2} \mathrm{O}$ en pacientes con SDRA moderado y de 15 a $20 \mathrm{mmH}_{2} \mathrm{O}$ en quienes cursen con SDRA severo. ${ }^{17}$

\section{Efectos sistémicos de la PEEP}

Efecto de PEEP en el estado hemodinámico y sistema cardiovascular: es importante destacar que no existe «PEEP fisiológico», lo que se tiene es una capacidad residual funcional, que junto con el factor surfactante que producen los neumocitos tipo II y el nitrógeno a nivel alveolar, evitan el colapso alveolar en respiración fisiológica; sin embargo, cuando el paciente se encuentra en estatus de ventilación mecánica, el diafragma y la caja torácica sufren disminución en su movilidad y, por lo tan- 


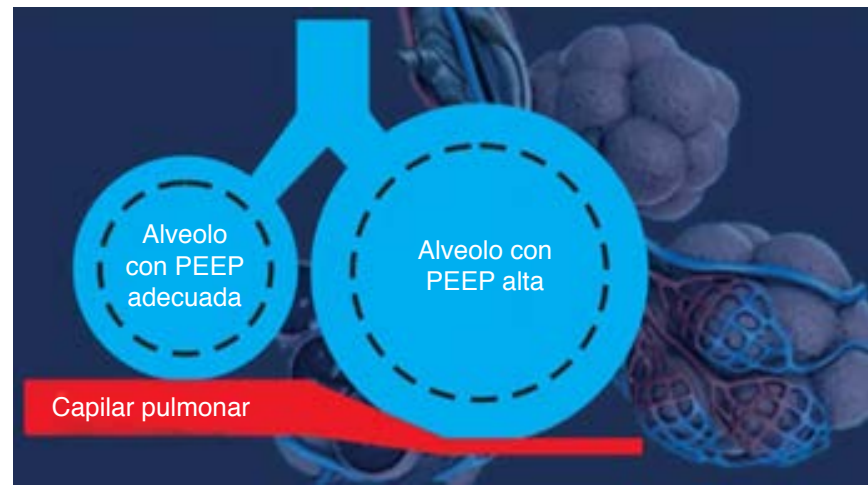

Figura 3: Sobredistensión alveolar con colapso de los capilares alveolares adyacentes.

to, la posibilidad de colapso alveolar es mayor; la programación de una «PEEP estándar» entre 5 a $8 \mathrm{cmH}_{2} \mathrm{O}$ en la mayoría de los casos tendrá efectos benéficos pulmonares y efectos cardiovasculares específicos (Figura 1).

La PEEP es un componente importante de la presión media de la vía aérea $(\mathrm{Pm})$, que se encuentra en estrecha relación con el trabajo ventricular derecho (VD), al aumentar la presión media, se aumenta la postcarga del VD, la cual si se mantiene por tiempo prolongado, puede conducir a falla ventricular derecha, y el aumento de la presión torácica con cada insuflación de presión positiva puede disminuir el retorno venoso, disminuyendo la precarga de las cavidades derechas, ambos componentes pueden comprometer la hemodinamia del paciente (Figura 2), conduciendo a choque obstructivo impuesto por el ventilador y pudiendo llevar a falla multiorgánica, principal causa de muerte en pacientes con SDRA severo. ${ }^{18}$

La ventilación con presión positiva afecta la precarga, postcarga y distensibilidad ventricular. El efecto sobre la precarga influye en una disminución del retorno de la sangre venosa hacia el corazón, por ende tendrá disminución del gasto cardiaco; para funcionar normalmente el ventrículo derecho debe generar la suficiente presión para vencer la suma de la presión de la arteria pulmonar y la presión positiva al final de la espiración, en consecuencia, si el PEEP es demasiado alto el ventrículo derecho comenzará a fallar debido a un aumento en la postcarga, el efecto neto será causar una disminución en el gasto cardiaco. ${ }^{19} \mathrm{El}$ efecto sobre la distensibilidad ventricular izquierda está determinado por el desplazamiento del tabique interventricular hacia la izquierda, lo que causa disminución en el área de superficie del ventrículo izquierdo con incapacidad para distenderse, presenta disminución en el llenado y por consecuencia disminución del gasto cardiaco (Figura 2). ${ }^{20}$

Efecto de PEEP en el sistema respiratorio: el ajustar un nivel alto de PEEP tiene por objetivo mantener abiertos los alveolos previamente reclutados, abiertos, pero por otra parte, tomando en cuenta que el daño pulmonar es heterogéneo en el SDRA, los alveolos que presentan menor daño o intactos pueden generar daño por sobredistensión, ${ }^{21,22}$ causado por la tensión secundaria a la presión positiva otorgada por el ventilador mecánico, contamos con distintas formas para evaluar esta característica; en la curva de presión-volumen se puede observar un «pico horizontal» cuando se llega al máximo de presión, que no es correspondido por un aumento proporcional del volumen, indicándonos que existe presión positiva sobre el alveolo, que no le genera ganancia de volumen, únicamente tensión; se han realizado estudios de tomografía por impedancia eléctrica para determinar cuál es la PEEP óptima para el paciente, en la cual se observan los alveolos colapsados, alveolos abiertos reclutados y alveolos sobredistendidos, lo cual puede ayudarnos a seleccionar un nivel de PEEP que genere la mayor apertura alveolar, con el menor grado de sobredistensión. ${ }^{23,24}$

La sobredistensión alveolar generada por PEEP alta puede condicionar colapso de los vasos capilares adyacentes a las unidades alveolares, contribuyendo al aumento de las resistencias vasculares pulmonares y pudiendo generar hipertensión pulmonar inducida por el ventilador mecánico (Figura 3). La presión de distensión alveolar (DP, por sus siglas en inglés driving pressure) es la diferencia entre la presión meseta y la PEEP y representa la tensión cíclica a la que se somete el parénquima pulmonar durante cada ciclo ventilatorio, aumentar el nivel de PEEP puede disminuir la DP al aumentar la presión transpulmonar y mantener los alveolos reclutados, así como la disminución de la DP demuestra un aumento de la Cest del paciente. ${ }^{25,26}$

La posición prono aumenta la posibilidad de supervivencia de los pacientes con SDRA (Figura 4), ${ }^{27}$ quienes responden a la maniobra aumentan significativamente la presión transpulmonar y la Cest; se ha observado en

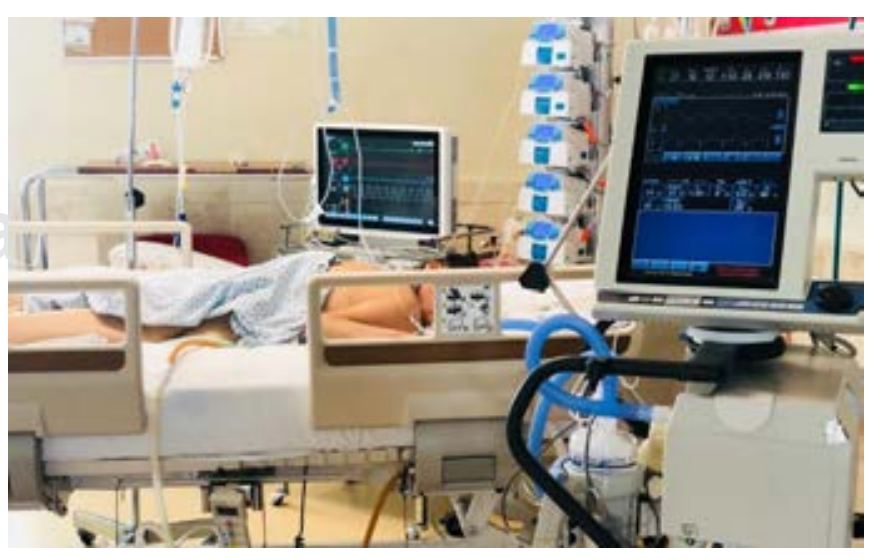

Figura 4: Paciente con síndrome de dificultad respiratoria aguda en posición prono. 


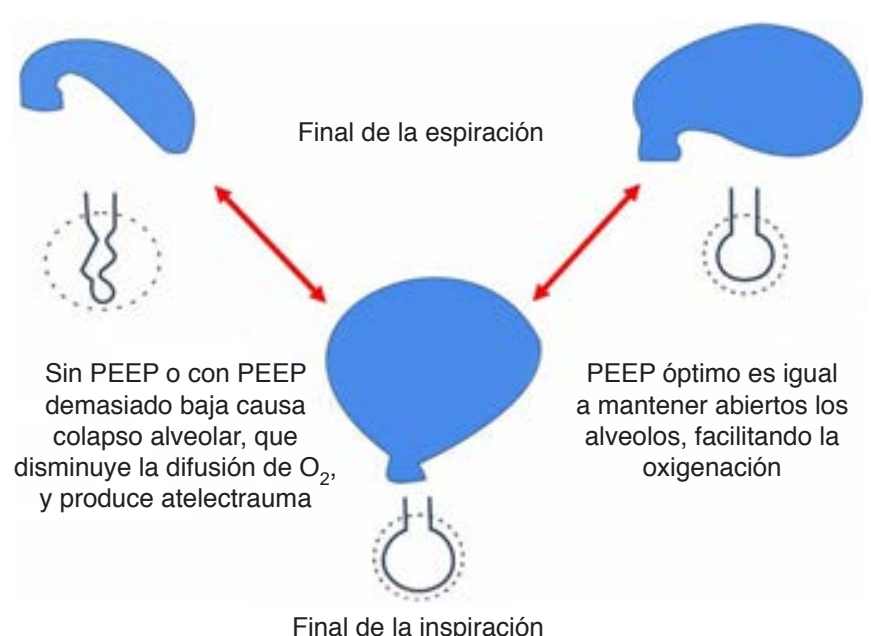

Figura 5: Efecto del PEEP sobre las atelectasias.

pacientes con SDRA severo que cuentan con monitoreo de la presión esofágica, ${ }^{28}$ que al iniciar la posición prono el aumento de la presión transpulmonar es suficiente, como para disminuir la PEEP, siempre y cuando se mantenga positiva la presión transpulmonar espiratoria, siendo una medida objetiva y útil para guiar su descenso o evaluar si requiere aumentar nuevamente el nivel de PEEP en cualquier momento que se vuelva negativa, lo que significaría la presencia de atelectrauma. Sin embargo, guiar el nivel de PEEP por catéter de presión esofágica no ha demostrado beneficio significativo en pacientes con SDRA. ${ }^{29,30}$

Efecto de PEEP en síndromes pleuropulmonares: frecuentemente en el paciente bajo ventilación mecánica con o sin SDRA se complican factores no pulmonares que disminuyen la distensibilidad pulmonar, ${ }^{31}$ tales como derrame pleural, las atelectasias o el neumotórax, lo cual puede modificar la necesidad de aumentar o descender la PEEP al menos de forma transitoria.

En la presencia de derrame pleural, se ha descrito que se debe interpretar adecuadamente la mecánica ventilatoria y colocar un nivel de PEEP suficiente para prevenir el colapso masivo de unidades alveolares y evitar un reclutamiento-desreclutamiento cíclico, ${ }^{32}$ por lo cual, si está indicado el drenaje y se resuelve, podría considerarse descender la PEEP a un nuevo nivel óptimo.

Se debe tratar de prevenir atelectasias con un PEEP óptimo, pero en el caso de formación de atelectasias, el aumento de PEEP únicamente aumentaría el espacio muerto de la vía respiratoria, aumentando el riesgo de sobredistensión en los alveolos menos afectados, al resolverse esta complicación, podría plantearse disminuir y optimizar el nivel de PEEP para prevenir atelectasias posteriores (Figura 5). ${ }^{33}$

El neumotórax iatrogénico por barotrauma, ${ }^{34} \mathrm{com}$ plicación cada vez menos frecuente en pacientes con
SDRA, debido a las medidas de protección pulmonar, puede aumentar proporcionalmente con la PEEP o favorecer a que se perpetúe esta situación, lo cual debe tomarse en cuenta para valorar tla colocación de una sonda o catéter pleural y cuándo es prudente retirarla, pese a que clínicamente se encuentre resuelta esta complicación.

Efectos de PEEP sobre el diafragma (miotrauma): el diafragma es el músculo primario encargado de la inspiración, con una función esencial para el mantenimiento de una ventilación adecuada, especialmente cuando la carga respiratoria es elevada. Existen cuatro determinantes de la fuerza del músculo respiratorio: 1) la fuerza contráctil, que incluye la longitud-tensión de la fibra muscular; 2) tasa de estimulación neural; 3) velocidad de acortamiento; y 4) curvatura del diafragma. Sobre esta última, se ve involucrada la presión positiva al final de la espiración debido a que, al aplicar niveles altos, la curvatura diafragmática se aplana y por ende el radio aumenta y la fuerza disminuye (ley de Laplace) (Figura 6). Se ha propuesto que el aumento de niveles altos de PEEP influyen de manera negativa sobre la estructura y función diafragmática ocasionando mayor disfunción diafragmática, llevando a mayores días de ventilación mecánica y con mayor incapacidad para la liberación.

Efectos de PEEP sobre presión intraabdominal y perfusión de órganos abdominales: la hipertensión intraabdominal (HIA $)^{35,36}$ se define como una presión intraabdominal (PIA) sostenida por arriba o igual a 12 $\mathrm{mmHg}$,una patología que pasa desapercibida por muchas unidades de cuidados intensivos, que aumenta la mortalidad directamente proporcional al grado de HIA, asociándose con un gasto cardiaco reducido por aumento de la resistencia vascular sistémica y

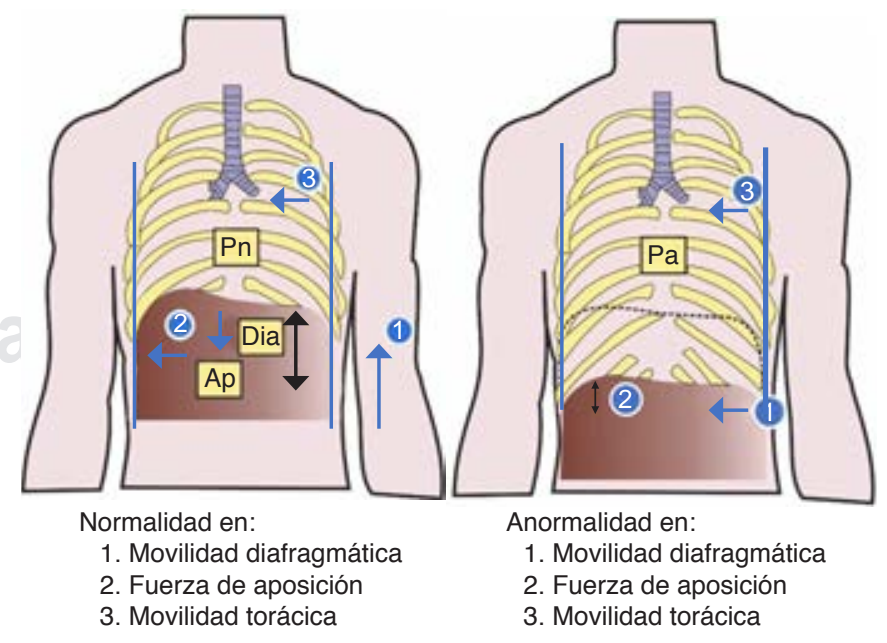

Figura 6: Miotrauma ocasionado por el PEEP. Se observa aplanamiento y acortamiento de las fibras musculares del diafragma. $\mathrm{Pn}=\mathrm{PEEP}$ normal, $\mathrm{Pa}=\mathrm{PEEP}$ alto, Dia = diafragma, $\mathrm{Ap}=$ aposición. 
Tabla 1: Recomendaciones sobre la presión positiva al final de la espiración y la presión intraabdominal.

\begin{tabular}{|c|c|c|c|c|}
\hline PIA & Normal & SDRA & HIA & $\mathrm{HIA}$ e IR \\
\hline $\begin{array}{l}\text { Titulación del } \\
\text { PEEP }\end{array}$ & $\begin{array}{l}\text { Se sugiere evitarlo por un exce- } \\
\text { so de presión de distensibilidad }\end{array}$ & $\begin{array}{l}\text { Optimizar la complianza respi- } \\
\text { ratoria; la DP más baja posible } \\
\text { con Vt protector }\end{array}$ & $\begin{array}{l}\text { Optimizar la complianza } \\
\text { respiratoria; la DP más baja } \\
\text { posible con Vt protector. Se } \\
\text { sugiere PEEP en } \mathrm{cmH}_{2} \mathrm{O}=\mathrm{PIA} \\
\text { en } \mathrm{mmHg}\end{array}$ & $\begin{array}{l}\text { Optimizar la complianza respi- } \\
\text { ratoria; la DP más baja posible } \\
\text { con Vt protector } \\
\text { Guiarse por presión esofágica } \\
\text { es una alternativa }\end{array}$ \\
\hline
\end{tabular}

$\mathrm{PIA}=$ presión intraabdominal, SDRA = síndrome de distrés respiratorio agudo, $\mathrm{HIA}=$ hipertensión intraabdominal, $\mathrm{IR}=$ insuficiencia respiratoria, $\mathrm{PEEP}=$ presión positiva al final de la espiración, DP = presión de distensibilidad (driving pressure), Vt = volumen tidal.

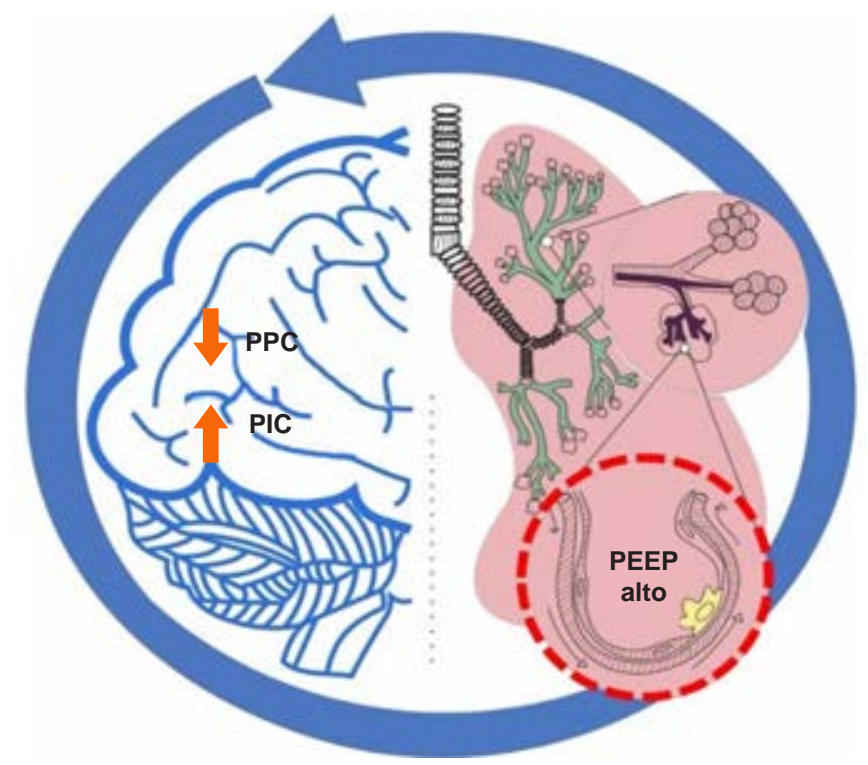

Figura 7: Efecto directo del PEEP sobre el encéfalo. Por arriba de 15 $\mathrm{cmH}_{2} \mathrm{O}$, disminuye la presión de perfusión cerebral (PPC) y aumenta la presión intracraneal (PIC), comprometiendo la dinámica cerebral.

una disminución del retorno venoso. ${ }^{37}$ Los pacientes SDRA pueden desarrollar patologías abdominales secundarias, asociadas con el aumento de la presión intraabdominal y viceversa, los problemas abdominales primarios pueden asociarse con hipertensión intraabdominal y secundariamente presentar SDRA.

La PIA elevada, junto con el gasto cardiaco alterado, reduce el flujo sanguíneo a órganos intraabdominales vitales, como los riñones y el hígado. ${ }^{38}$

La ventilación mecánica se considera un factor independiente para el aumento de la PIA, especialmente cuando se aplica presión positiva al final de la espiración (PEEP) o en presencia de auto-PEEP, tal como lo demostró un estudio multicéntrico observacional de 100 pacientes donde se incluyeron pacientes con ventilación mecánica y pacientes sin ventilación mecánica, se realizaron dos mediciones al día de la PIA, se consideró
HIA si la medición era igual o superior a $12 \mathrm{mmHg}$. Los resultados de los pacientes bajo ventilación mecánica fue media $6.7 \pm 4.1 \mathrm{mmHg}$ y significativamente mayor que en pacientes sin ventilación mecánica $3.6 \pm 2.4$ $\mathrm{mmHg}(p=0.0001){ }^{39}$

La PEEP alta también se ha asociado con congestión hepática debida a la disminución del flujo venoso portal y arterial hepático generado por la impedancia venosa con disminución del retorno venoso a consecuencia de la presión intratorácica alta de forma sostenida, y actualmente se identifica el uso de PEEP como un factor predisponente para la elevación de la PIA que puede disminuir la presión de perfusión abdominal (PPA) y, por consecuencia, disminución de la presión de perfusión renal, lo que conlleva disminución en el filtrado glomerular y oliguria hasta anuria, debido al aumento del péptido natriurético auricular y aumento de la hormona antidiurética. ${ }^{40}$

El aumento de la PIA trae como consecuencia restricción de los movimientos de la caja torácica por descenso del diafragma y disminución de la capacidad pulmonar total y disminución de la distensibilidad pulmonar, también se asocia con atelectasias con aumento de las presiones transdiafragmáticas, las presiones inspiratorias de las vías respiratorias y la elastancia de la pared torácica. ${ }^{41}$ En el 2019 Regli y colaboradores ${ }^{42}$ realizaron un estudio donde ofrecen recomendaciones sobre el paciente con PIA elevada y su abordaje en la ventilación, que van desde garantizar una medición adecuada de la PIA e iniciar manejo médico (1C), hasta realizar una laparotomía descompresiva (1D) (Tabla 1).

Efecto de PEEP sobre los capilares linfáticos: el SDRA se caracteriza fisiopatológicamente por una pérdida de tejido pulmonar aireado como resultado del acúmulo de edema alveolar y la producción de atelectasia, lo que reduce la distensibilidad pulmonar y altera el intercambio de gases. Al aplicar PEEP como un intento de apertura pulmonar, puede tener efectos negativos en el drenaje del edema alveolar, la dinámica de los fluidos en el intersticio pulmonar se regula 
por presiones internas y externas de los capilares, la matriz extracelular y los linfáticos pulmonares, y difiere entre la respiración espontánea y la ventilación mecánica, en condiciones normales los vasos linfáticos recogen líquidos a través de tres vías: hiliar, transpleural y transabdominal a través de presión negativa en el intersticio. ${ }^{43} \mathrm{El}$ aumento de la presión en los alvéolos, con un aumento de la presión inspiratoria, media o al final de la espiración, puede alterar notablemente la función de los linfáticos, lo que determina una reducción en la capacidad de drenaje del líquido durante la respiración espontánea, la presión en el intersticio es más alta que la presión en los linfáticos, lo que produce un gradiente negativo (alrededor de $3-4 \mathrm{mmHg}$ ) que facilita el drenaje continuo de líquidos. ${ }^{44}$ Por el contrario, durante la presión positiva, se produce un aumento en las presiones intersticiales y linfáticas, en un grado similar $(10 \mathrm{mmHg})$. En este caso, el gradiente entre el intersticio y los linfáticos se vuelve alrededor de cero o incluso positivo, lo que perjudica el posible drenaje de líquidos. La ventilación mecánica con PEEP más alta afecta negativamente el drenaje linfático desde el pulmón, lo que posiblemente perjudica el intercambio de líquido del tejido pulmonar intersticial. ${ }^{45}$
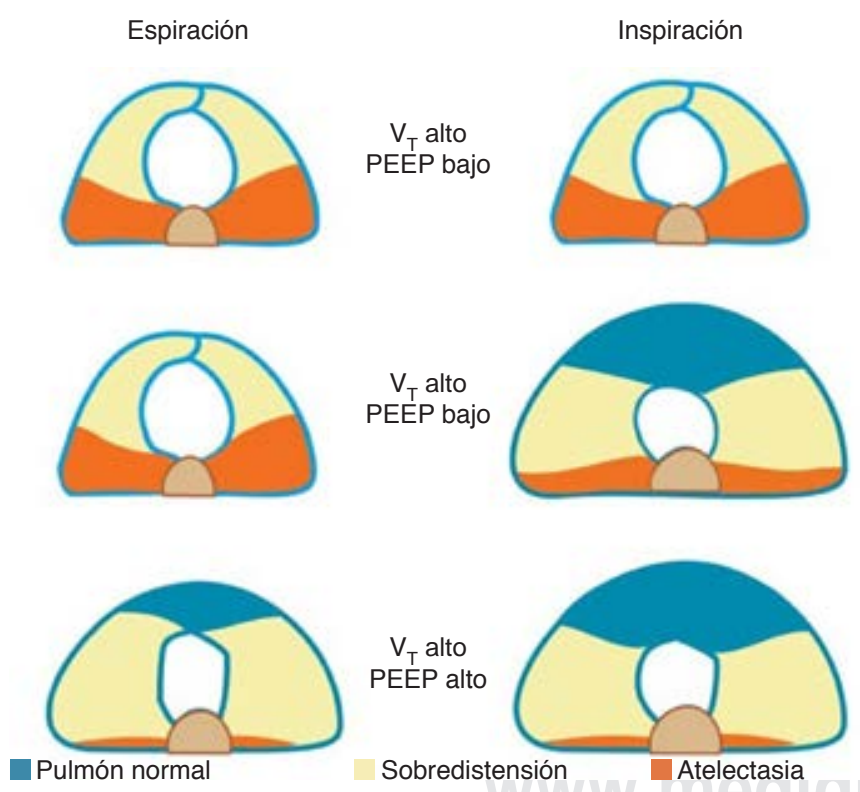

Figura 8: Efecto del PEEP y volumen tidal sobre los pulmones durante la anestesia general. Regiones pulmonares atelectasias (naranja), regiones pulmonares infladas en exceso (azules), regiones normalmente aireadas (blancas). Durante la VM con Vt bajo y PEEP baja, se presentan mayores atelectasias en la espiración y áreas mínimas de sobreinflación en la inspiración. En la VM con Vt alto y PEEP baja, hay menos atelectasias presentes al final de la inspiración, con áreas aumentadas de sobreinflación en la inspiración. Con VM bajo Vt alto y PEEP bajo, hay menos atelectasias al final de la espiración e inspiración, con áreas de sobreinflación en la inspiración. Modificada de: Güldner A, et al. ${ }^{46}$

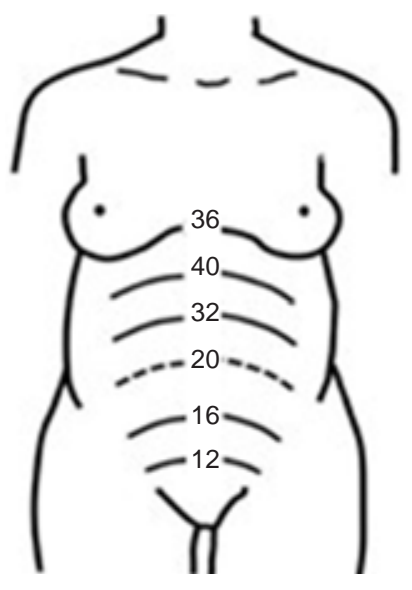

Efecto de PEEP sobre la presión intracraneal: la hemodinamia cerebral representa el comportamiento de la dinámica cerebral ante los cambios que presenta la fisiología que envuelve la economía, mediante la presión de perfusión cerebral (PPC), que se encuentran estrechamente relacionados con el comportamiento de la presión intracraneal (PIC), PPC: PAM-PIC, la evidencia médica disponible recomienda por consenso de expertos, conservar la PPC de $60-160 \mathrm{mmHg}$ para mantener la autorregulación cerebral. ${ }^{47}$

Koutsokou y colaboradores realizaron un estudio en pacientes neurocríticos sin SDRA, con dos grupos; en un grupo se aplicaba 0 PEEP (ZEEP) y a otro PEEP de $8 \mathrm{cmH}_{2} \mathrm{O}$, no hubo repercusión en la PPC ni PIC, pero en el grupo ZEEP hubo más complicaciones pulmonares. 48

Caricato y su equipo de investigadores evaluaron la influencia directa del PEEP sobre la hemodinamia cerebral, aplicando PEEP desde $0,5,8,12 \mathrm{cmH}_{2} \mathrm{O}$, demostrando que con un PEEP de $12 \mathrm{cmH}_{2} \mathrm{O}$ disminuía la PAM, incrementaba la PVC, pero era transitoria sin efecto deletéreo, con un beneficio de incremento de la Crs correlacionada directamente con PIC. ${ }^{49}$ Muench y colaboradores realizaron un estudio donde elevan el PEEP hasta $20 \mathrm{cmH}_{2} \mathrm{O}$, notando una considerable disminución de la PAM, reduciendo el FSC y elevando la PIC. ${ }^{50}$ La PEEP alta también condiciona un incremento de la presión intracraneal, niveles cercanos a $15 \mathrm{cmH}_{2} \mathrm{O}$ de PEEP se relacionan con un aumento significativo de la presión intracraneal (Figura 7), por lo que en pacientes con cráneo hipertensivo se coloca una PEEP alta debe hacerse de forma juiciosa con monitoreo de PIC continua. ${ }^{51}$

Efecto del PEEP en la sala de operaciones: en el quirófano un gran porcentaje de cirugías requerirán de intubación y una programación adecuada de la ventilación mecánica para protección pulmonar y un pilar en esto es 
la presión positiva al final de la espiración, hay que tomar en cuenta diversos factores: las comorbilidades que presenta el paciente en su ingreso a cirugía, el tipo de cirugía (abdominal, torácica, laparoscópica, extremidades, neurocirugía, etcétera), posición del paciente (supino, prono, sentado decúbito lateral, etcétera), la ventilación mecánica per se durante el transanestésico representa una serie de cambios sobre la dinámica ventilatoria, como disminución de la capacidad residual funcional, distensibilidad pulmonar, desplazamiento del diafragma, cambios en la zonas de West, etcétera. ${ }^{52}$ EI PEEP ideal en anestesia es un área gris, existe una serie de recomendaciones, para evitar complicaciones pulmonares postoperatorias (CPP) y daño inducido por el ventilador; sin embargo, ${ }^{53}$ (Figura 8) las recomendaciones varían en iniciar ZEEP hasta $7 \mathrm{cmH}_{2} \mathrm{O}$ para una persona con un IMC normal, para un IMC de 25-30 iniciar con PEEP de 8-10 $\mathrm{cmH}_{2} \mathrm{O} .{ }^{46}$ El estudio IMPROVE comparó dos grupos: uno con estrategia no protectora $(10-12 \mathrm{~mL} / \mathrm{kg}$ peso predicho [PP] con 0 PEEP) versus otro grupo con estrategia protectora (6-8 mL/kg PP y 6-8 $\mathrm{cmH}_{2} \mathrm{O}$ ) con maniobras de reclutamiento (MR), observando disminución de las complicaciones pulmonares postoperatorias en el grupo de ventilación protectora. ${ }^{54}$ PROVHILO comparó 0-2 vs $12 \mathrm{cmH}_{2} \mathrm{O}$ de PEEP más MR con el mismo Vt 6-8 $\mathrm{mL} / \mathrm{kg}$ de PP para los dos grupos, reportando no disminución de incidencias en las CPP en ambos grupos; sin embargo, más efectos deletéreos hemodinámicos en el grupo de alto PEEP. ${ }^{55}$ LAS VEGAS un estudio observacional, su media de comparación fue PEEP de 3.5-5 $\mathrm{cmH}_{2} \mathrm{O}$, sin encontrarse asociación de CPP en esta colocación de PEEP. ${ }^{56}$ El estudio PROBESE, un estudio multicéntrico con pacientes obesos (IMC $\geq 35$ ) que serían sometidos a cirugía laparoscópica o no, con duración mayor a dos horas, incluyó dos grandes grupos: los dos grupos con Vt de $7 \mathrm{~mL} / \mathrm{kg}$ de PP; el primer grupo con PEEP alto de $12 \mathrm{cmH}_{2} \mathrm{O}$ con MR y el segundo grupo con PEEP de $4 \mathrm{cmH}_{2} \mathrm{O}$ sin MR, en ambos grupos las CPP fueron similares, se pudo observar que el grupo de bajo PEEP presentó más hipoxemia y en el de alto PEEP la hipotensión y bradicardia fueron más comunes. ${ }^{57}$ Sobre las MR para abrir el pulmón, sólo bajo hipoxemia refractaria considerando riesgos y beneficios, en cirugías de urgencia, desde el inicio hasta la emersión de la anestesia se mantuvo a pacientes con PEEP de 6-8 $\mathrm{cmH}_{2} \mathrm{O}$, resultando en menor incidencia de CPP hablándonos del beneficio del PEEP durante el transanestésico. ${ }^{58}$ Con base en esto podemos recomendar durante la anestesia, iniciar con bajo PEEP $5 \mathrm{cmH}_{2} \mathrm{O}$ e ir tomando la decisión de modificarlo durante el acto quirúrgico de acuerdo con los requerimientos del paciente, acompañarlo de medidas de protección pulmonar, no recomendamos iniciar con PEEP altos y no realizar MR a menos que estén indicadas.

Efecto de la PEEP en la obesidad: la obesidad es una epidemia que ha aumentado exponencialmente, convirtiéndose en un problema mundial de salud. ${ }^{59}$ Es hoy en día bastante común el ingreso a cirugía o $\mathrm{UCI}$ de un paciente con obesidad, se estima que $20 \%$ de los casos admitidos a la UCI presentan obesidad. ${ }^{60,61}$ El paciente obeso tiene características diferentes a uno con IMC normal o bajo, caracterizado por efectos negativos sobre la pared torácica debido a la masa presentada en la pared torácica y abdominal, afectando directamente la distensibilidad, volumen pulmonar al final de la espiración disminuido, capacidad residual funcional disminuida, aumento de posibilidad de cierre de la vía aérea, formación de atelectasias, disminución en la oxigenación arterial, así como alteraciones de la mecánica pulmonar y ventilatoria. Por lo que se debe ajustar su presión meseta acorde a su PIA.

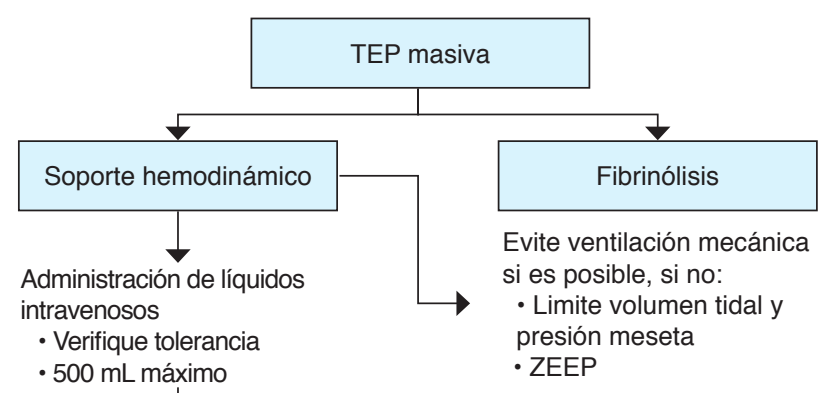

Figura 10: Abordaje integral del paciente con tromboembolia pulmonar. Evitar la intubación, si es necesaria no agregar PEEP.

Tabla 2: Recomendaciones sobre el uso de presión positiva al final de la espiración en el paciente obeso.

\begin{tabular}{|c|c|c|c|}
\hline Programación inicial & Metas en la VMC & Vigilancia del daño por VMC & Maniobras de Rescate \\
\hline
\end{tabular}

$\mathrm{VMC}=$ ventilación mecánica controlada, $\mathrm{Vt}=$ volumen tidal, $\mathrm{Fr}=$ frecuencia respiratoria, $\mathrm{Pr}=$ presión, $\mathrm{SDRA}=$ síndrome de distrés respiratorio agudo, $\mathrm{PEEP}=$ presión al final de la espiración, $\mathrm{PP}$ = peso predicho, $\mathrm{MR}$ = maniobras de reclutamiento, $\mathrm{ECMO}$ = circulación extracorpórea por membrana de oxigenación. 
Tabla 3: Cambios fisiológicos en la vía aérea/ventilación de la embarazada.

\begin{tabular}{|c|c|c|}
\hline Parámetro & Cambios & Impacto en la ventilación \\
\hline $\begin{array}{l}\text { Vías respiratorias } \\
\text { altas }\end{array}$ & $\begin{array}{l}\text { Mucosas con edema por } \\
\text { hipervascularidad }\end{array}$ & $\begin{array}{l}\text { Se considera vía aérea } \\
\text { difícil y regularmente } \\
\text { requieren uso de tubo endo } \\
\text { traqueal de menor calibre a } \\
\text { lo habitual }\end{array}$ \\
\hline Diafragma & $\begin{array}{l}\text { Restricción secundaria a } \\
\text { útero grávido }\end{array}$ & $\begin{array}{l}\text { Disminución de la capa- } \\
\text { cidad residual funcional } \\
\text { pulmonar; hipoxia por } \\
\text { periodos de apnea }\end{array}$ \\
\hline Estómago & $\begin{array}{l}\text { Motilidad de tubo digestivo } \\
\text { reducida y aumento de la } \\
\text { presión intragástrica }\end{array}$ & $\begin{array}{l}\text { Aumento de la probabili- } \\
\text { dad de broncoaspiración }\end{array}$ \\
\hline
\end{tabular}

Presión meseta ajustada para obesidad $=$ Pr meseta + (presión intraabdominal - 13) / 2.

La meta en paciente obeso con SDRA es presión meseta ajustada $<27 \mathrm{cmH}_{2} \mathrm{O}$, la presión de distensión pulmonar puede ser considerada mayor a la meta para paciente no obeso, debido a que parte de esta presión está destinada a distender la caja torácica y el abdomen del paciente, repercutiendo ligeramente menos sobre las paredes alveolares, la meta de presión de distensión en este tipo de pacientes debe ser $<17 \mathrm{cmH}_{2} \mathrm{O}$ en un paciente con SDRA, tomando en cuenta la influencia del PEEP sobre la fórmula (presión meseta-PEEP), se recomienda iniciar con ventilación protectora, por peso predicho en pacientes con SDRA e ideal en pacientes con no SDRA, PEEP moderado a alto y si es requerido $M R,{ }^{50}$ no se recomienda en paciente con no SDRA Vt bajo. Mientras el PEEP previene el colapso y optimiza la vía aérea, es asociado con inestabilidad hemodinámica, su eso en el paciente obeso es debatido. Pacientes obesos con pulmones sanos recomiendan un PEEP moderado de 5-8 $\mathrm{cmH}_{2} \mathrm{O}$ y considerar el uso de la guía de bajo PEEP ARDSNetwork para el paciente obeso, con un límite alto de $17 \mathrm{cmH}_{2} \mathrm{O}$ en pacientes con SDRA y $15 \mathrm{cmH}_{2} \mathrm{O}$ en pacientes con no SDRA, ${ }^{62}$ vigilando nuestra DP (un incremento del PEEP no debe estar asociado con elevación de la DP). ${ }^{63-65} \mathrm{En}$ la Tabla 2 observamos las recomendaciones del uso de PEEP en el paciente obeso.

\section{Embarazo}

La paciente obstétrica presenta una serie de cambios fisiológicos propios del embarazo en todo su organismo, es muy importante determinar la altura del fondo uterino (Figura 9), ya que esto ocasionará una cefalización de los hemidiafragmas, disminuyendo así la capacidad residual funcional, los cambios más importantes en la vía aérea y ventilación se mencionan en las Tablas 3 y 4.
Se debe tener en cuenta que la embarazada se considera vía aérea difícil cuatro veces más que pacientes sin embarazo, y se considera con estómago lleno, por lo que se deben prever métodos alternativos para el manejo avanzado de la vía aérea, en caso de intubación orotraqueal considere tubo endotraqueal diámetro pequeño (\#7 en promedio).

La paciente obstétrica que requiere de ventilación mecánica es infrecuente ${ }^{66}$ se recomienda un volumen tidal 6-8 $\mathrm{mL} / \mathrm{kg}$ de PP, un PEEP de 5-8 $\mathrm{cmH}_{2} \mathrm{O}$ (algo similar a lo que recomienda Pelosi en pacientes obesos pulmonarmente sanos) ${ }^{67,68}$ y mantener en metas de protección pulmonar, siendo aplicables las guías de ventilación mecánica para paciente no embarazada. ${ }^{69}$

\section{ZEEP}

La maniobra de ZEEP (Zero PEEP), es una actitud que se debe de tomar en ciertos escenarios clínicos, pero antes que nada veamos el porqué, con base en estas dos preguntas: ¿existe el PEEP fisiológico? No, la presión pleural negativa va de $-2 \mathrm{a}-10 \mathrm{cmH}_{2} \mathrm{O}$, esto de acuerdo con la altura y a la posición, generando un volumen residual, que en realidad es un concepto diferente. ¿Qué ofrece el PEEP al alveolo? La presión positiva al final de la espiración funciona como una férula/stent neumático de las generaciones bronquiales más distales y el hecho de no colocarle PEEP al paciente bajo ventilación mecánica tiene más contras que pros. Se utilizó el ZEEP en pacientes quirúrgicos en diversos estudios, ${ }^{70}$ siendo superior en oxigenación y en disminución de complicaciones pulmonares postoperatorias en los grupos con PEEP, la técnica PEEP-ZEEP, asistida posteriormente por una compresión torácica en fisioterapia pulmonar es una maniobra para ayudar con el manejo de secreciones. ${ }^{71}$ En los escenarios críticos donde el PEEP ofrezca

Tabla 4: Cambios fisiológicos de la vía aérea/ventilación en el embarazo y su impacto en la reanimación.

\begin{tabular}{|c|c|c|}
\hline Parámetro & Cambios & Impacto en la ventilación \\
\hline $\begin{array}{l}\text { Volumen minuto } \\
\text { pulmonar }\end{array}$ & $\uparrow 45 \% / \downarrow \mathrm{PCO}_{2}$ & $\begin{array}{l}\text { Disminución de la } \\
\text { capacidad de amortiguar; } \\
\text { presentan acidosis como } \\
\text { mecanismo compensador a } \\
\text { la alcalosis respiratoria }\end{array}$ \\
\hline $\begin{array}{l}\text { Aporte de oxígeno } \\
\left(\mathrm{DO}_{2}\right)\end{array}$ & $\uparrow 20 \%$ & $\begin{array}{l}\text { Pueden presentar rápida- } \\
\text { mente hipoxia tisular ante } \\
\text { inestabilidad hemodinámica } \\
\text { o respiratoria }\end{array}$ \\
\hline $\begin{array}{l}\text { Capacidad residual } \\
\text { funcional }\end{array}$ & $\downarrow 25 \%$ & \\
\hline $\begin{array}{l}\text { Distensibilidad } \\
\text { dinámica }\end{array}$ & $\begin{array}{l}\downarrow \text { Secundaria a útero } \\
\text { grávido que desplaza el } \\
\text { diafragma, disminuyendo } \\
\text { así la presión transalveolar }\end{array}$ & \\
\hline
\end{tabular}


más riesgo que beneficio es donde se debe de tomar esta actitud, en aquéllas donde genere presión al borde del colapso o la falla hemodinámica, como lo son la falla ventricular derecha, hay que recordar que el PEEP ofrece una presión sobre el alveolo y puede repercutir directamente sobre el VD, ${ }^{72,73}$ tromboembolia pulmonar (TEP), esto debido a que el PEEP incrementa la sobrecarga del VD, por lo que debe evitarse incluso la intubación en el paciente con TEP (Figura 10), ${ }^{74}$ éstas son dos indicaciones del ZEEP, existen puntos controversiales como el paciente con hipertensión pulmonar severa y fallo ventricular izquierdo con choque cardiogénico, ${ }^{75}$ hay que recordar las dos indicaciones puntuales de ZEEP; TEP y fallo del VD, siempre individualizar al paciente y recordar las dos preguntas iniciales: ¿existe el PEEP fisiológico? y ¿qué ofrece el PEEP al alveolo?

\section{PEEP en el paciente pediátrico}

El paciente pediátrico comprende una serie de diferencias en la vía aérea en comparación con el adulto en tamaño, forma, posición, colapsabilidad de la vía aérea, que hasta los ocho años ya representa características similares a las del adulto. ${ }^{76,77}$ Se recomienda el uso de PEEP para evitar el colapso al- veolar, en pacientes pulmonarmente sanos se recomienda iniciar con un PEEP de $3-5 \mathrm{cmH}_{2} \mathrm{O}$, PEEP moderado con paciente con daño pulmonar limitado y en pacientes con daño pulmonar severo un PEEP puede que sea necesario (siempre es importante individualizar al paciente), siempre se debe buscar el PEEP que ofrezca mejor oxigenación sin repercusiones hemodinámicas. Se recomienda PEEP alto para estabilizar y evitar el colapso en pacientes que se encuentren con traqueo o broncomalacia. No se recomiendan las maniobras de reclutamiento rutinarias a menos que presenten indicación. ${ }^{78}$ En pacientes con SDRA se recomienda adherirse a las recomendaciones de PEEP y metas de oxigenación del PARDS; $\mathrm{SpO}_{2}$ 92-97\%, con un PEEP $<10 \mathrm{cmH}_{2} \mathrm{O}$ y $88-92 \%$ con un $\mathrm{PEEP} \geq 10 \mathrm{cmH}_{2} \mathrm{O} .{ }^{79,80}$

\section{DISCUSIÓN}

Inmediatamente después de intubar a un paciente y aplicarle ventilación mecánica con presión positiva intermitente estamos obligados a preguntarnos ¿cuándo retiraremos la ventilación mecánica? y al colocar un nivel de PEEP, debemos preguntarnos si el paciente presenta alguna patología pulmonar o en donde el PEEP no le ofrezca algún beneficio, hemos podido observar

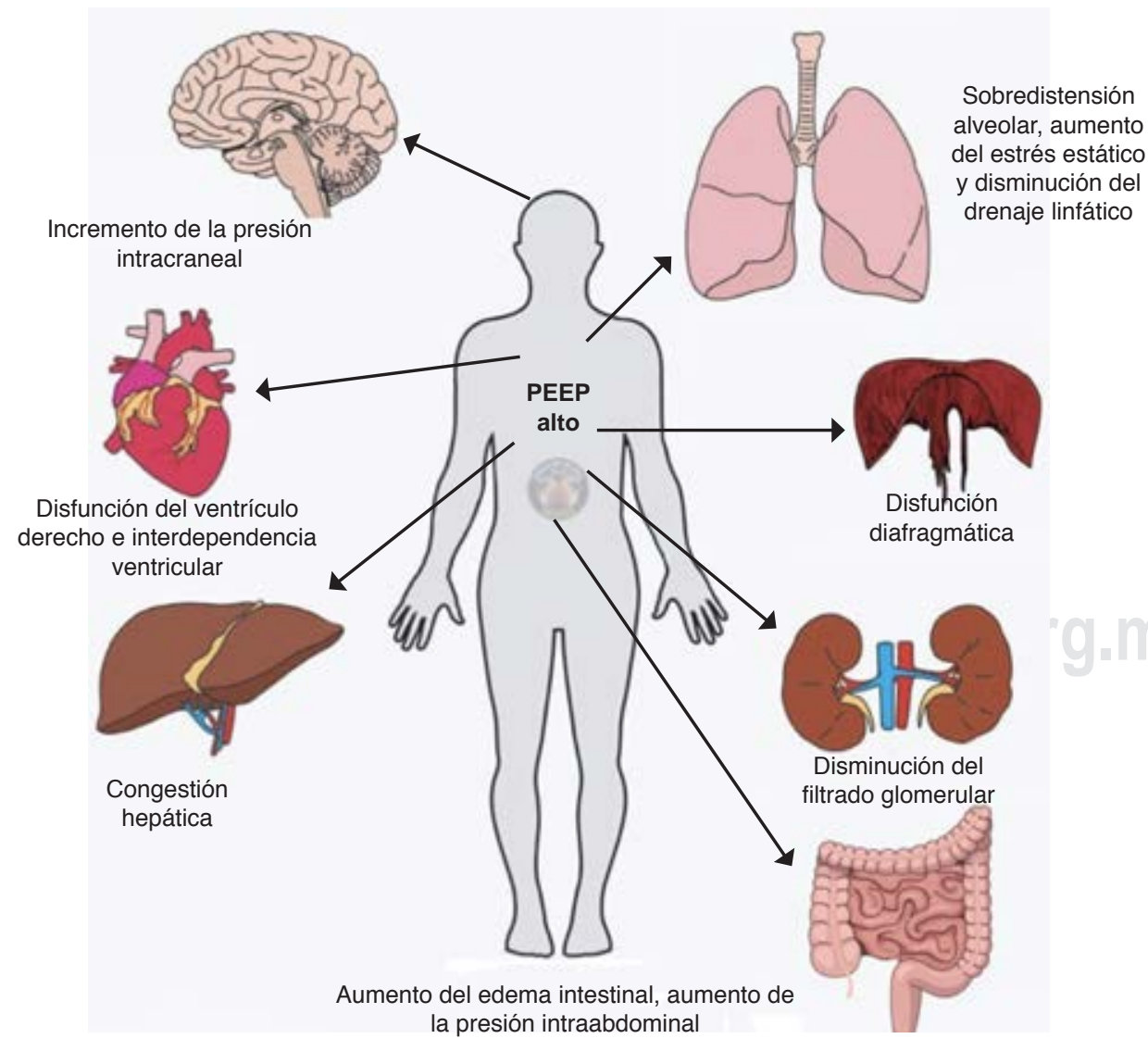

Figura 11:

Efectos deletéreos del PEEP alto sobre diversos órganos. 
Tabla 5: Recomendaciones sobre la PEEP en los distintos escenarios clínicos.

\begin{tabular}{|c|c|c|}
\hline Situación clínica & PEEP & Consideraciones \\
\hline $\begin{array}{l}\text { Obesidad e hipertensión } \\
\text { intrabdominal|36-38,42,57,62,63 }\end{array}$ & $\begin{array}{l}\text { Sin SDRA: } \\
\text { IMC > 30: iniciar } 8 \mathrm{cmH}_{2} \mathrm{O} \\
\text { IMC > 40: } 10 \mathrm{cmH}_{2} \mathrm{O} \text { o colocar de } 50 \text { a } \\
70 \% \text { de la PIA }\left(\mathrm{cmH}_{2} \mathrm{O}\right) \\
\text { Con SDRA, considere ajustar de acuerdo } \\
\text { a Tabla de PEEP/FiO } \text { de ARDSNetwork } \\
\text { considerando presión meseta ajustada } \\
\text { a PIA }\end{array}$ & $\begin{array}{l}\text { Malbrain y Pelosi proponen limitar presión meseta, calculando la presión meseta ajustada a la } \\
\text { hipertensión intraabdominal con la siguiente fórmula: } \\
\text { Presión meseta meta en } \mathrm{cmH}_{2} \mathrm{O}-7+0.7 \times \text { x PIA en mmHg } \\
\text { Considerar DP hasta de } 17 \mathrm{~cm} \mathrm{H}_{2} \mathrm{O} \\
\text { Considerar el uso de balón esofágico para mantener una presión espiratoria transpulmonar de } \\
1 \text { a } 10 \mathrm{~cm} \mathrm{H}_{2} \mathrm{O} \text { con una driving pressure transpulmonar }<15 \mathrm{~cm} \mathrm{H}_{2} \mathrm{O} \text { y una presión inspiratoria } \\
\text { transpulmonar }<27 \mathrm{~cm} \mathrm{H}_{2} \mathrm{O} \text {. Esta estrategia no ha demostrado disminución en mortalidad } \\
\text { Reducir PEEP en caso de hipotensión, sobredistensión, incremento de la DP o incremento de } \\
\text { la presión meseta }\end{array}$ \\
\hline $\begin{array}{l}\text { Síndromes } \\
\text { pleuropulmonares }\end{array}$ & $\begin{array}{l}\text { PEEP: } 5-8 \mathrm{cmH}_{2} \mathrm{O} \\
\text { Neumotórax a tensión: PEEP } 0 \mathrm{cmH}_{2} \mathrm{O} \\
\text { Atelectasias: } 5 \mathrm{cmH}_{2} \mathrm{O} \\
\text { Derrame pleural: hasta } 10 \mathrm{cmH}_{2} \mathrm{O}\end{array}$ & $\begin{array}{l}\left.\text { Ante colapso hemodinámico por neumotórax a tensión colocar ZEEP (PEEP de } 0 \mathrm{~cm} \mathrm{H}_{2} \mathrm{O}\right) \\
\text { hasta resolver } \\
\text { No incrementar PEEP para tratar atelectasias } \\
\text { Colocar mínimo necesario para prevenir atelectasias }\left(5 \mathrm{~cm} \mathrm{H}_{2} \mathrm{O}\right) \\
\text { Considerar disminuir PEEP al resolver el derrame pleural }\end{array}$ \\
\hline $\mathrm{SDRA}^{26}$ & $\begin{array}{l}\text { Utilizar la Tabla PEEP bajo/FiO }{ }_{2} \text { alto de } \\
\text { ARDSNetwork }\end{array}$ & 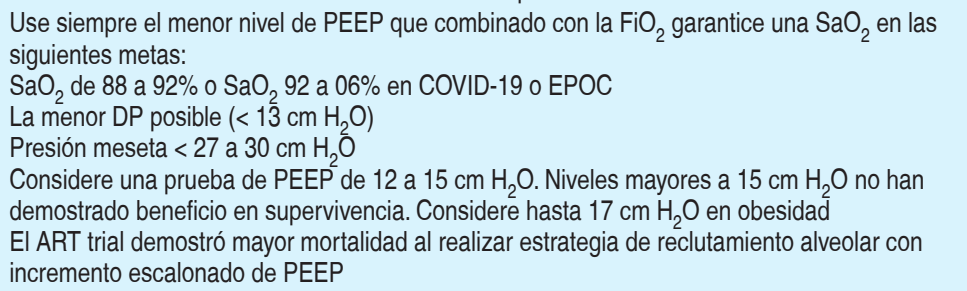 \\
\hline $\begin{array}{l}\text { Hipertensión } \\
\text { intracraneal }{ }^{46-49}\end{array}$ & PEEP: 5 a $8 \mathrm{cmH}_{2} \mathrm{O}$ & $\begin{array}{l}\text { En caso de ARDS concomitante, no colocar PEEP > } 10 \text { a } 15 \mathrm{cmH}_{2} 0 \text { si no se cuenta con } \\
\text { monitoreo de presión intracraneal }\end{array}$ \\
\hline Transoperatorio ${ }^{46,53-58}$ & PEEP: 5 a $8 \mathrm{cmH}_{2} \mathrm{O}$ & $\begin{array}{l}\text { No realizar MR rutinariamente } \\
\text { PEEP mínimo de } 4 \mathrm{~cm} \mathrm{H}_{2} \mathrm{O} \\
\text { En pacientes obesos se puede iniciar con } 8-10 \mathrm{cmH}_{2} \mathrm{O} \\
\text { En caso de hemorragia grave, considerar disminuir PEEP }\end{array}$ \\
\hline
\end{tabular}

PEEP = presión positiva al final de la espiración, VM = ventilación mecánica, LOV-ED = lung protective ventilation in the emergency department, IMC = índice de masa corporal, SDRA = síndrome de distrés respiratorio agudo, PIA = presión intraabdominal, DP = distensión alveolar, ZEEP = zero end expiration pressure, ART = alveolar recruitment for $A R D S$ trial, $\mathrm{MR}=$ maniobras de reclutamiento, $\mathrm{RCP}=$ respiración cardiopulmonar, $\mathrm{ROSC}$ = retorno de la circulación espontánea.

que en los diferentes casos, escenarios y estados patológicos se aborda de manera distinta su programación inicial del PEEP y su manejo durante su ventilación mecánica invasiva; desde el paciente pediátrico, embarazada, obeso, con falla cardiaca, neurocrítico, en el área de quirófano, etcétera, y sobre todo a un paciente con diagnóstico establecido de SDRA, debemos preguntarnos: ¿cuándo y cómo disminuiremos la PEEP?

Colocar un nivel de PEEP es una estrategia útil para evitar la hipoxemia, pero puede causar barotrauma en los pulmones y estrés estático, así como efectos depresores en el corazón, el destete o liberación de la PEEP es una parte integral del retiro de la ventilación mecánica, pero para la selección de pacientes, la reducción y el monitoreo apropiado no se ha establecido cuándo se colocan niveles altos de PEEP, lo ideal sería disminuirlos lo más rápido posible sin sacrificar la oxigenación; sin embargo, no existe una guía hasta el día de hoy de cómo bajar la PEEP de forma ideal.

La PEEP no es inocua y tiene complicaciones específicas en varios órganos y sistemas (Figura 11), tales como deterioro a la eliminación del $\mathrm{CO}_{2}$, aumento de la postcarga al ventrículo derecho, la cual si se prolonga por un tiempo considerable puede llevar al paciente a presentar falla cardiaca derecha aguda que, si los niveles de PEEP son muy elevados, conllevan desplazamiento del tabique interventricular de derecha a izquierda, lo que trae como consecuencia disminución del llenado del ventrículo izquierdo y por ende falla ventricular izquierda con disminución del gasto cardiaco. 


\section{CONCLUSIONES}

La presión positiva al final de la espiración ofrece una ventilación pulmonar protectora al realizar un trabajo, evitando el colapso alveolar. Hay múltiples patologías que requieren de ventilación mecánica invasiva, en la cual debemos realizar una protección alveolar, donde el PEEP es parte fundamental; existen diversos tipos de programar el PEEP inicial, no se recomienda iniciar con un PEEP alto a excepción de sujetos con SDRA severo, ni realizar maniobras de reclutamiento a excepción de que presente alguna indicación (Tabla 5). La PEEP representa una serie de cambios en la economía del paciente, por lo tanto, a la hora de colocarla debemos conocer muy bien sus cambios sobre el sistema cardiovascular, respiratorio, linfático, cerebro y el impacto directo sobre el diafragma, pudiendo crear un miotrauma. En los pacientes con HIA es muy importante la adecuada colocación del PEEP partiendo desde $5 \mathrm{cmH}_{2} \mathrm{O}$ y valorando si presenta SDRA para realizar cambios en este inicio de titulación. El paciente neurocrítico presenta una PEEP mayor a $15 \mathrm{cmH}_{2} \mathrm{O}$ y crea disminución de la PPC y aumento de la PIC, por lo que no se recomiendan PEEP alto de inicio. En sujetos obesos representa un reto por la disminución en la elasticidad pulmonar, si el paciente no presenta SDRA iniciar con $5 \mathrm{cmH}_{2} \mathrm{O}$ e ir buscando el PEEP óptimo. En el área de quirófano recomendamos iniciar con PEEP de 4-8 $\mathrm{cm} \mathrm{H}_{2} \mathrm{O}$, individualizando al paciente, posición y tipo de cirugía; aunque sea obeso no se recomienda iniciar con PEEP alto y tampoco con 0 de PEEP, esto para evitar CPP y evitar episodios de hipoxemia, tampoco recomendamos MR sin indicación. En paciente obstétrica, por los cambios fisiológicos que representa el embarazo, se recomienda un PEEP inicial de 4-8 $\mathrm{cmH}_{2} \mathrm{O}$ e ir en búsqueda de la optimización. En paciente pediátrico sin daño pulmonar hay que iniciar con un PEEP de 3-5 $\mathrm{cmH}_{2} \mathrm{O}$, evitar los PEEP altos, a menos que presente PARDS severo, en donde buscaremos mejorar la hipoxemia y un PEEP de $10 \mathrm{cmH}_{2} \mathrm{O}$. El ZEEP solamente representa indicación en los escenarios del casos con fallo del VD y cuando presente TEP. En resumen, la mayoría de la programación del PEEP inicial es con PEEP bajos, evitar iniciar con PEEP alto, ZEEP solamente para los pacientes mencionados, no recomendamos MR rutinariamente o sin indicación, la PEEP óptima es aquélla que ofrece una mejor oxigenación y no representa cambios hemodinámicos sobre su economía.

\section{REFERENCIAS}

1. Barach AL, Martin J, Eckman M. Positive pressure respiration and its application to the treatment of acute pulmonary edema. Ann Intern Med. 1938;12:754-795.
2. Cournand A, Motley HL, Werko L. Mechanism underlying cardiac output change during intermittent positive pressure breathing (IPP). Fed Proc. 1947;6:92.

3. Ashbaugh DG, Bigelow DB, Petty TL, et al. Acute respiratory distress in adults. Lancet. 1967;2:319-323.

4. Suter PM, Fairley B, Isenberg MD. Optimum end expiratory airway pressure in patients with acute pulmonary failure. $N$ Engl J Med. 1975;292:284-289.

5. Tenaillon A, Labrousse J, Gateau O, et al. Optimal positive endexpiratory pressure and static lung compliance. $N$ Engl J Med. 1978;299:774-775.

6. Dantzker DR, Lynch JP, Weg JG. Depression of cardiac output is a mechanism of shunt reduction in the therapy of acute respiratory failure. Chest. 1980;77:636-642.

7. Rapin M, Lemaire F, Regnier B, et al. Increase of intrapulmonary shunting induced by dopamine. Proc R Soc Med. 1977;70 Suppl 2:71-75.

8. Lemaire F, Harf A, Simonneau G, et al. Gas exchange, static pressure-volume curve and positive-pressure ventilation at the end of expiration. Study of 16 cases of acute respiratory insufficiency in adults. Ann Anesthesiol Fr. 1981;22:435-441.

9. Mead J, Takishima T, Leith D. Stress distribution in lungs: a model of pulmonary elasticity. J Appl Physiol. 1970;28:596-608.

10. Lachmann B. Open up the lung and keep the lung open. Intensive Care Med. 1992;18:319-321.

11. Bouferrache K, Vieillard-Baron A. Acute respiratory distress syndrome, mechanical ventilation, and right ventricular function. Curr Opin Crit Care. 2011;17:30-35.

12. Manual básico de ventilación mecánica AVENTHO. Ed. Prado; Cap. $11 ; 163-165$.

13. Kacmarek RM, Villar J, Sulemanji D, Montiel R, Ferrando C, Blanco $\mathrm{J}$, et al. Open lung approach for the acute respiratory distress syndrome: a pilot, randomized controlled trial. Crit Care Med. 2016;44(1):32-42.

14. Sánchez Casado M, Quintana Díaz M, Palacios D, Hortigüela V, Marco Schulke C, García J, et al. Relationship between the alveolar-arterial oxygen gradient and $\mathrm{PaO}_{2} / \mathrm{FiO}_{2}$, introducing peep into the model. Med Intensiva. 2012;36(5):329-334.

15. Biasi A, Aranha E, Nasi L, De Moraes D, Petri L, Penna H, et al. Effect of lung recruitment and titrated positive end-expiratory pressure (PEEP) vs low PEEP on mortality in patients with acute respiratory distress syndrome a randomized clinical trial. JAMA. 2017; 318 (14): 1335-1345. doi: 10.1001/jama.2017.14171.

16. Pérez Nieto OR, Zamarrón López El, Soriano Orozco R, Guerrero Gutiérrez MA, Morgado Villaseñor LA, Sánchez Díaz JS, et al. Síndrome de distrés respiratorio agudo: abordaje basado en evidencia. Intensive Qare. 2019. doi: 10.13140/ RG.2.2.26627.96800.

17. Vieillard-Baron A, Matthay M, Teboul JL, et al. Experts' opinion on management of hemodynamics in ARDS patients: focus on the effects of mechanical ventilation. Intensive Care Med. 2016;42:739-749.

18. Jardin F, Vieillard-Baron A. Right ventricular function and positive pressure ventilation in clinical practice: from hemodynamic subsets to respirator settings. Intensive Care Med. 2003;29(9):1426-1434.

19. Luecke T, Pelosi P. Clinical review: positive end-expiratory pressure and cardiac output. Crit Care. 2005;9(6):607-621.

20. van der Zee P, Gommers D. Recruitment maneuvers and higher PEEP, the so-called open lung concept, in patients with ARDS. Crit Care. 2019;23(1):73.

21. Amini R, Herrmann J, Kaczka DW. Intratidal overdistention and derecruitment in the injured lung: a simulation study. IEEE Trans Biomed Eng. 2017;64(3):681-689.

22. Zhao Z, Chang MY, Chang MY, Gow CH, Zhang JH, Hsu YL, et al. Positive end-expiratory pressure titration with electrical impedance tomography and pressure-volume curve in severe acute respiratory distress syndrome. Ann Intensive Care. 2019;9(1):7.

23. Sribar A, Merc V, Persec Z, Persec J, Milas I, Husedzinovic $S$. Influence of different PEEP levels on electrical impedance tomography findings in patients under general anesthesia ventilated in the lateral decubitus position. J Clin Monit Comput. 2020;34(2):311-318 . 
24. Bugedo G, Retamal J, Bruhn A. Driving pressure: a marker of severity, a safety limit, or a goal for mechanical ventilation? Crit Care. 2017;21(1):199. doi: 10.1186/s13054-017-1779-x.

25. Amato MB, Meade MO, Slutsky AS, et al. Driving pressure and survival in the acute respiratory distress syndrome. $N$ Engl J Med. 2015;372:747-755.

26. Guérin C, Reignier J, Richard JC, Beuret P, Gacouin A, Boulain T, PROSEVA study group. Prone positioning in severe acute respiratory distress syndrome. NEJM. 2013;368(23):2159-2168.

27. Piraino T, Cook D. Optimal PEEP guided by esophageal balloon manometry. Respiratory Care. 2011;56(4):510-513.

28. Calvancanti A, Amato M, Serpa-Neto A. The elusive search for "best PEEP" and whether esophageal pressure monitoring helps. JAMA. 2019;321(9):839-841.

29. Akoumianaki E, Maggiore SM, Valenza F, Bellani G, Jubran A, Loring SH, et al. The application of esophageal pressure measurement in patients with respiratory failure. $A m \mathrm{~J}$ Respir Crit Care Med. 2014;189(5):520-531.

30. Formenti P, Graf J, Santos A, Gard KE, Faltesek K, Adams AB, et al. Non-pulmonary factors strongly influence the stress index. Intensive Care Med. 2011;37(4):594-600.

31. Graf J, Formenti P, Santos A, Gard K, Adams A, Tashjian J, et al. Pleural effusion complicates monitoring of respiratory mechanics. Crit Care Med. 2011;39(10):2294-2299.

32. Rama-Maceiras P. Atelectasias perioperatorias y maniobras de reclutamiento alveolar. Arch Bronconeumol. 2010;46(6):317-324.

33. Hsu CW, Sun SF. latrogenic pneumothorax related to mechanical ventilation. World J Crit Care Med. 2014;3(1):8-14.

34. Lindqvist $J$, van den Berg $M$, van der Pijl R, Hooijman PE, Beishuizen A, Elshof J. Positive end-expiratory pressure ventilation induces longitudinal atrophy in diaphragm fibers. $A m$ J Respir Crit Care Med. 2018;198(4):472-485.

35. Kirkpatrick AW, Roberts DJ, De Waele J, et al. Intra-abdominal hypertension and the abdominal compartment syndrome: updated consensus definitions and clinical practice guidelines from the World Society of the Abdominal Compartment Syndrome. Intensive Care Med. 2013;39:1190-1206.

36. Reintam Blaser A, Regli A, De Keulenaer B, et al. Incidence, risk factors, and outcomes of intra-abdominal hypertension in critically ill patients-a prospective multicenter study (IROI study). Crit Care Med. 2019;47:535-542.

37. Regli A, Mahendran R, Fysh ET, Roberts B, Noffsinger B, De Keulenaer BL, et al. Matching positive end-expiratory pressure to intra-abdominal pressure improves oxygenation in a porcine sick lung model of intra-abdominal hypertension. Crit Care. 2012;16(5):R208.

38. Soler Morejón Cde D, Tamargo Barbeito TO. Effect of mechanical ventilation on intra-abdominal pressure in critically ill patients without other risk factors for abdominal hypertension: an observational multicenter epidemiological study. Ann Intensive Care. 2012;2 Suppl 1(Suppl 1):S22.

39. Jacob LP, Chazalet JJ, Payen DM, Villiers SM, Boudaoud S, Teillac $P$ et al. Renal hemodynamic and functional effect of PEEP ventilation in human renal transplantations. Am J Respir Crit Care Med. 1995;152(1):103-107.

40. Torquato JA, Lucato JJ, Antunes T, Barbas CV. Interaction between intra-abdominal pressure and positive-end expiratory pressure. Clinics (Sao Paulo). 2009;64(2):105-112.

41. Regli A, Pelosi P, Malbrain MLNG. Ventilation in patients with intra-abdominal hypertension: what every critical care physician needs to know. Ann Intensive Care. 2019;9(1):52.

42. Fan E, Del Sorbo L, Goligher EC, Hodgson CL, Munshi L, Walkey AJ, et al. An Official American Thoracic Society/ European Society of Intensive Care Medicine/Society of Critical Care Medicine Clinical Practice Guideline: mechanical ventilation in adult patients with acute respiratory distress syndrome. $\mathrm{Am} \mathrm{J}$ Respir Crit Care Med. 2017;195(9):1253-1263.

43. Malbrain M, Pelosi P. Open up and keep the lymphatics open: they are the hydraulics of the body! Crit Care Med. 2006;34(11):2860-2862.

44. Moriondo A, Mukenge S, Negrini D. Transmural pressure in rat initial subpleural lymphatics during spontaneous or mechanical ventilation. Am J Physiol Heart Circ Physiol. 2005;289(1):H263-H269.
45. Pelosi P, Rocco PRM, Gama de Abreu M. Close down the lungs and keep them resting to minimize ventilator-induced lung injury. Crit Care. 2018;22(1):72.

46. Güldner A, Kiss T, Serpa Neto A, Hemmes SN, Canet J, Spieth $\mathrm{PM}$, et al. Intraoperative protective mechanical ventilation for prevention of postoperative pulmonary complications: a comprehensive review of the role of tidal volume, positive end-expiratory pressure, and lung recruitment maneuvers. Anesthesiology. 2015;123(3):692-713.

47. Rodríguez Boto G, Rivero Garvia M, Gutiérrez González R, Márquez Rivas J. Conceptos básicos sobre la fisiopatología cerebral y la monitorización de la presión intracraneal. Neurología. 2015;30(1):16-22.

48. Koutsoukou A, Perraki H, Raftopoulou A, et al. Respiratory mechanics in braindamaged patients. Intensive Care Med. 2006;32(12):1947-1954.

49. Caricato A, Conti G, Della Corte F, Mancino A, Santilli F, Sandroni C, et al. Effects of PEEP on the intracranial system of patients with head injury and subarachnoid hemorrhage: the role of respiratory system compliance. J Trauma. 2005;58(3):571576.

50. Ball L, Serpa Neto A, Pelosi P. Obesity and survival in critically ill patients with acute respiratory distress syndrome: a paradox within the paradox. Crit Care. 2017;21(1):114.

51. Muench E, Bauhuf $C$, Roth $H$, et al. Effects of positive endexpiratory pressure on regional cerebral blood flow, intracranial pressure, and brain tissue oxygenation. Crit Care Med. 2005;33(10):2367-2372.

52. Borsellino B, Schultz MJ, Gama de Abreu M, Robba C, Bilotta F. Mechanical ventilation in neurocritical care patients: a systematic literature review. Expert Rev Respir Med. 2016;10(10):11231132.

53. Manual básico de Ventilación mecánica AVENTHO. Ed. Prado; Cap. 1; 1-8.

54. Pelosi P, Hedenstierna G, Ball L, Edmark L, Bignami E. The real role of the PEEP in operating room: pros \& cons. Minerva Anestesiol. 2018;84(2):229-235.

55. Futier E, Constantin JM, Paugam-Burtz C, Pascal J, Eurin M, Neuschwander A, et al. A trial of intraoperative low-tidal-volume ventilation in abdominal surgery. N Engl J Med. 2013;369:428-437.

56. PROVE Network Investigators for the Clinical Trial Network of the European Society of Anaesthesiology, Hemmes SN, Gama de Abreu M, Pelosi P, Schultz MJ. High versus low positive end-expiratory pressure during general anaesthesia for open abdominal surgery (PROVHILO trial): a multicentre randomised controlled trial. Lancet. 2014;384(9942):495-503.

57. The LAS VEGAS investigators. Epidemiology, practice of ventilation and outcome for patients at increased risk of postoperative pulmonary complications: LAS VEGAS: an observational study in 29 countries. Eur J Anaesthesiol. 2017;34:492-507.

58. Writing Committee for the PROBESE Collaborative Group of the PROtective VEntilation Network (PROVEnet) for the Clinical Trial Network of the European Society of Anaesthesiology, Bluth T, Serpa Neto A, Schultz MJ, Pelosi P, Gama de Abreu $M$ et al. Effect of intraoperative high positive end-expiratory pressure (PEEP) with recruitment maneuvers vs low PEEP on postoperative pulmonary complications in obese patients: a randomized clinical trial. JAMA. 2019;321(23):2292-2305. doi: 10.1001/jama.2019.7505. Erratum in: JAMA. 2019;322(18):18291830.

59. Hedenstierna G, McCarthy GS. Airway closure and closing pressure during mechanical ventilation. Acta Anaesthesiol Scand. 1980;24:299-304.

60. Hales CM, Fryar CD, Carroll MD, et al. Differences in obesity prevalence by demographic characteristics and urbanization level among adults in the United States, 2013-2016. JAMA. 2018;319:2419-2429.

61. Pepin JL, Timsit JF, Tamisier R, et al. Prevention and care of respiratory failure in obese patients. Lancet Respir Med. 2016;4:407-418.

62. De Jong A, Molinari N, Pouzeratte $\mathrm{Y}$, et al. Difficult intubation in obese patients: incidence, risk factors, and complications in 
the operating theatre and in intensive care units. $\mathrm{Br} J$ Anaesth. 2015;114:297-306.

63. De Jong A, Chanques G, Jaber S. Mechanical ventilation in obese ICU patients: from intubation to extubation. Crit Care. 2017;21:63.

64. Ball L, Pelosi P. How I ventilate an obese patient. Crit Care. 2019;23:176.

65. Neto AS, Hemmes SN, Barbas CS, et al. Association between driving pressure and development of postoperative pulmonary complications in patients undergoing mechanical ventilation for general anaesthesia: a metaanalysis of individual patient data. Lancet Respir Med. 2016;4(4):272-280.

66. Chestnut D, Wong C, Tsen L, Ngan Kee WD, Jill Mhyre YB, et al. Chestnut's obstetric anesthesia: principles and practice. Chapter 2; Physiologic changes of pregnancy. 6th edition. Elsevier; 2019. pp. 15-38.

67. Munnur U, Bandi VD, Gropper MA. Airway management and mechanical ventilation in pregnancy. In: Rosene-Montella K, Bourjeily G (eds). Pulmonary problems in pregnancy. Respiratory medicine. Humana Press; 2009. pp. 385-403.

68. Munnur U, Karnad DR, Bandi VD, et al. Critically ill obstetric patients in an American and an Indian public hospital: comparison of case-mix, organ dysfunction, intensive care requirements, and outcomes. Intensive Care Med. 2005;31(8):1087-1094.

69. Casey E, Hayes N, Ross A, Connolly L, Dob D, Zimmerman J. Obstetric critical care, clinical problems. ESICM. 2013.

70. Muthu V, Agarwal R, Dhooria S, Prasad KT, Aggarwal AN, Suri $V$, et al. Epidemiology, lung mechanics and outcomes of ARDS: a comparison between pregnant and non-pregnant subjects. $J$ Crit Care. 2019;50:207-212.

71. Lapinsky SE, Rojas-Suarez JA, Crozier TM, Vasquez DN, Barrett $\mathrm{N}$, Austin K, et al. Mechanical ventilation in critically-ill pregnant women: a case series. Int J Obstet Anesth. 2015;24(4):323-328.

72. Barbosa FT, Castro AA, de Sousa-Rodrigues CF. Positive endexpiratory pressure (PEEP) during anaesthesia for prevention of mortality and postoperative pulmonary complications. Cochrane Database Syst Rev. 2014;(6):CD007922.

73. Oliveira ACO, Lorena DM, Gomes LC, Amaral BLR, Volpe MS. Effects of manual chest compression on expiratory flow bias during the positive end-expiratory pressure-zero end-expiratory pressure maneuver in patients on mechanical ventilation. $J$ Bras Pneumol. 2019;45(3):e20180058.

74. Haddad F, Doyle R, Murphy DJ, Hunt SA. Right ventricular function in cardiovascular disease, part II: pathophysiology, clinical importance, and management of right ventricular failure. Circulation. 2008;117:1717-1731.

75. Moitra VK, Einav S, Thies KC, Nunnally ME, Gabrielli A, Maccioli $\mathrm{GA}$, et al. Cardiac arrest in the operating room: resuscitation and management for the anesthesiologist: Part 1. Anesth Analg. 2018;126(3):876-888.

76. Meyer G, Vieillard-Baron A, Planquette B. Recent advances in the management of pulmonary embolism: focus on the critically ill patients. Ann Intensive Care. 2016;6(1):19.

77. Garrido Galindo C, Flores Hernández SS, Pérez Redondo $\mathrm{CN}$. Diferencias anatomofuncionales y endoscópicas entre la vía aérea del niño y la del adulto. Rev Inst Nal Enf Resp Mex. 2007;2:142-148.

78. Kneyber MCJ, de Luca D, Calderini E, Jarreau PH, Javouhey E, Lopez-Herce J, et al. Recommendations for mechanical ventilation of critically ill children from the Paediatric Mechanical Ventilation Consensus Conference (PEMVECC). Intensive Care Med. 2017;43(12):1764-1780.

79. Rimensberger PC, Cheifetz IM; Pediatric Acute Lung Injury Consensus Conference Group. Ventilatory support in children with pediatric acute respiratory distress syndrome: proceedings from the pediatric acute lung injury consensus conference. Pediatr Crit Care Med. 2015;16(5 Suppl 1):S51-S60.

80. Khemani RG, Smith L, Lopez-Fernandez YM, Kwok J, Morzov $\mathrm{R}$, Klein MJ, et al. Paediatric acute respiratory distress syndrome incidence and epidemiology (PARDIE): an international, observational study. Lancet Respir Med. 2019;7(2):115-128.

\author{
Correspondencia: \\ Dr. Manuel Alberto Guerrero Gutiérrez \\ Médico Residente de 1er año en Medicina \\ Crítica del Instituto Nacional de Cancerología. \\ E-mail: manuelguerreromd@gmail.com
}

